\title{
Brain teaser
}

How does the brain work? How does a storm of electrical and biochemical chaos taking place within a few pounds of tissue underlie thought and calculation, memory and emotion, functions we cannot replicate even with our best technologies? This year, two new 'grand' scientific projects - the US BRAIN Initiative and the European Commission's Human Brain Project have set out to answer these questions. The challenge is immense, as is the range of sciences required: from psychology and molecular biology to laser physics and information theory.

But real progress is likely to depend on improvements in measurement. There are, as a recent Nature editorial noted (Nature 499, 253; 2013), about as many neurons in the human brain as there are pages on the Web. Yet the interconnectivity in the brain is far richer, with each neuron linking to thousands of others in a nonlinear way. We still don't know what the structure of the brain looks like. And beyond architecture, brain function depends on delicate correlations in the behaviour of millions of neurons right across the brain. Our ability to monitor and record these detailed dynamics remains limited.

Although the technology for recording neural activity with wire electrodes has advanced consistently for decades doubling in resolution every seven years since the 1950s - we can still only observe the dynamics of at most a few hundred neurons on timescales fast enough (milliseconds) to capture their core behaviour. Optical microscopy can get readings on something like 100,000 neurons, but only every second or so. Magnetic resonance imaging allows non-invasive whole-brain recordings on a one-second timescale, but doesn't come close to resolving the activity of single neurons.

By analogy, we can see inside the brain now in roughly the same way that astronomers could see into outer space a few hundred years ago. With early optical telescopes, scientists could explore the Solar System and such things as comets and nebulae, but deeper knowledge of everything from galaxies to black holes awaited other methods to make the invisible visible, including radio, X-ray and gamma-ray astronomy. If the ambitious brain projects do make spectacular progress, it will probably be due to a host of new technologies that will do the same for the human brain, creating

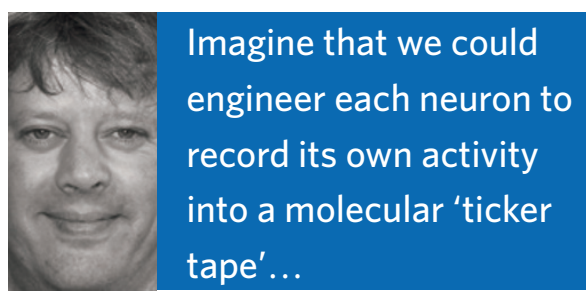

vastly more powerful ways to record neural activity - using anything from laser light to DNA.

Which will ultimately be the best? No one knows, of course. But physicist Adam Marblestone and colleagues have recently tried to look at how some of the current trends in technology might play out in the future (preprint at http://arxiv.org/abs/1306.5709; 2013). One conclusion: recording from every neuron isn't as out of reach as you might think.

Any suitable brain-imaging technology must avoid interference with normal function, both in terms of the power that can be dissipated within the brain, and the alteration of the physical tissue through direct influence. Both limits restrict the scope for traditional electrode technologies, even with the development of ever thinner wires and smaller electrode impedances. Hence, the biggest advances will probably come from radically different technologies.

An already well-developed alternative is to use light, in particular by attaching fluorescent indicator proteins to neurons. The light emitted varies with the neural state and can be detected outside the brain to read out neural patterns. This technique is now about ten times too slow to capture spiking activity with millisecond resolution, and also suffers from the fact that the brain is far from transparent to visible light; for example, scattering obscures signals coming from neurons deep in the brain.

A promising alternative is to use multiphoton techniques, which rely on infrared laser excitation and require the stimulation of a fluorescent protein through the near-simultaneous absorption of two laser-generated photons. Using this technique, researchers can now easily follow neural activity as far as $1 \mathrm{~mm}$ below the surface of animal brains. Unfortunately, the power dissipated by the intense lasers required is still far too large to permit wholebrain imaging without damaging the tissue.

But all this so far is just physics, more or less, determined by the properties of light and materials. More exciting possibilities emerge if one thinks of nanotechnology. Why not embed tiny electronic devices all through the brain to gather and digitize neural data, and then beam that information outside on radio waves?

As the authors point out, today's technology already allows the manufacture of wirelessly powered radiofrequency identification chips as small as $50 \mu \mathrm{m}$ in diameter, and chip-integrated antennae about ten times that size. Reduce these dimensions a little further and cells such as macrophages may be able to ingest such devices and deliver them throughout the brain. The idea of recording information from every one of the neurons in the brain starts to become conceivable.

This is still a dream, not least because today's microelectronic devices consume 100 to 1,000 times too much power for use in whole-brain monitoring with millisecond resolution.

Most exciting, perhaps, is the idea of using biology itself to gather neural information. Imagine that we could engineer each neuron to record its own activity into a molecular 'ticker tape' - a book made of biological macromolecules. One idea for doing this involves engineering a DNA polymerase to have an error rate sensitive to neural activity, perhaps linked to membrane voltage, for example. A strand of DNA would then include a density of errors providing a faithful rendering of the history of the associated neuron.

Using genetic technology, such systems could record from all neurons at the same time. But not quite yet, of course. Making this kind of molecular recording work with millisecond temporal resolution demands significant advances in synthetic biology, including the creation of a fast polymerase with the required sensitivity to neural activity. Can this be done? It sounds crazy but not that crazy, really.

In their article, Marblestone and colleagues quote Freeman Dyson from his book Imagined Worlds, thinking about how a future technology might place enough tiny devices into the brain to record from each and every neuron. "There is", he noted, "no law of physics that declares such an observational tool to be impossible." This reality might be closer than we think.

MARK BUCHANAN 